Papers in Physics, vol. 10, ART. 100003 (2018)

www.papersinphysics.org

Received: 8 January 2018, Accepted: 11 January 2018

Edited by: A. Martí

Licence: Creative Commons Attribution 4.0

DOI: http://dx.doi.org/10.4279/PIP.100003

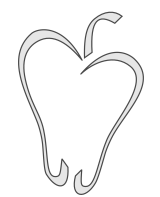

ISSN 1852-4249

\title{
Commentary on "Modeling temperature manipulation in a circular model of birdsong production"
}

\author{
Y. S. Zhang ${ }^{1 *}$
}

The paper titled "Modeling temperature manipulations in a circular model of birdsong production" by Dima et al. [1] suggests that the birdsong system has a circular architecture in which an initiating area in the brainstem provides inputs to both the downstream respiratory area and the upstream vocal control area; the latter subsequently sends input to the respiratory area as well. A consequence of the proposed architecture is that the birdsong syllables are generated by the neural commands at two different timescales corresponding to the two inputs. The model is successful in explaining the syllable stretching and breaking phenomena when an upstream vocal area is cooled down. In this commentary, I make some remarks on the findings of this paper and discuss how this work fits into the current knowledge about birdsong generation.

The avian vocal production system has long been understood as a feedforward network mainly implemented by a descending motor pathway. In this picture, a forebrain area HVC (high vocal center, as a proper name) generates the precisely timed sequential actions of this complex vocal behavior. The projection neurons in HVC fire sparsely at specific time points of the song. These activities activate the downstream RA (the robust nucleus of the arcopallium) where the motor patterns are generated by the activation of a subset of the RA neu-

\footnotetext{
*E-mail: yz9@princeton.edu

1 Princeton Neuroscience Institute, Princeton, 08544 New Jersey, USA.
}

rons. The RA neurons then activate the hypoglossal nerve (nXIIts) which controls the syringeal muscles and the respiratory group [2]. It has also been established that in addition to the descending pathway, there is an ascending pathway from the brainstem to HVC mediated by the nucleus Uva where the feedback from respiration is fed into HVC. The descending and ascending pathways form a loop [3].

The circular model proposed in Dima et al. [1] is compatible with the known architecture of the avian vocal system. Furthermore, the authors hypothesized that a slower timescale, independent of the moment-to-moment timing control from the HVC dynamics, exists in this architecture. In their model, this timescale is determined by the pulse activities of an initiating area (IA). This activity usually signals the start of a long syllable or a bout of brief pulses. The IA is thought to be in the brainstem which directly provides input to the expiratory related area (ER). Simultaneously, the IA also sends information to the $\mathrm{HVC} 2$ via the ascending pathway and initiates the HVC activities. With this additional degree of freedom in their model, they can simulate the respiratory patterns of all types of syllables present in canary songs. The setup is further supported by the HVC cooling effects on canary respiratory patterns during singing.

Whereas this study provides further understanding of the dynamics of the avian vocal production system, it depends on a number of assumptions that currently lack strong empirical evidence. One major assumption is the direct input from the hy- 
Papers in Physics, vol. 10, ART. 100003 (2018) / Y. S. Zhang

pothetical IA to the ER. Anatomically, the dorsal medial nucleus of the intercollicular complex (DM) seems to project to both the respiratory group and Uva. However, whether it behaves as the proposed model has not been reported. Second, the assumption that the syllable type determines the necessity of direct input seems a bit arbitrary. Why would the model undergo a qualitative change between different syllable types? Third, syllable types depend on very specific parameter settings of the IA activity. If we look at the spectrogram of the canary song shown in Fig. 1 of Ref. [1], the transformation from one type of syllable to another is rather gradual. However, the model-predicted IA activity exhibits quite distinct behaviors. As the model depends on several parameters, such prediction may be the result of overfitting.

One possible way to interpret the IA activity is that it may represent the sensory feedback from the respiratory system. The biomechanics of respiration imposes a strong constraint on the song dynamics [4]. It is conceivable that the slow timescale is originated from respiration. A future expansion of the circular model to perhaps a two-loop model with the respiratory system included may help understanding the origin and integration of the multiple timescales in the song production system.
In conclusion, this study provides a convincing evidence for the existence of multiple timescales in the avian song production system. On the one hand, it is compatible with the popular models that emphasize the role of $\mathrm{HVC}$ in timing control. On the other hand, it proposes alternative generators of song timing.

[1] G C Dima, M A Goldin, G B Mindlin, Modeling temperature manipulations in a circular model of birdsong production, Pap. Phys. 10, 100002 (2018).

[2] M S Fee, A A Kozhevnikov, R H Hahnloser, Neural mechanisms of vocal sequence generation in the songbird, Ann. NY. Acad. Sci. 1016, 1 (2004).

[3] R C Ashmore, J M Wild, M F Schmidt, Brainstem and forebrain contributions to the generation of learned motor behaviors for song, J. Neurosci. 25, 37 (2005)

[4] M F Schmidt, F Goller, Breathtaking songs: Coordinating the neural circuits for breathing and singing, Physiology 31, 6 (2016). 